\title{
Implications of Left Ventricular Dysfunction at Presentation for Infants with Coarctation of the Aorta
}

\author{
Carol A. McFarland ${ }^{1}$ (D) - Dongngan T. Truong ${ }^{1} \cdot$ Nelangi M. Pinto $^{1} \cdot$ L. LuAnn Minich ${ }^{1} \cdot$ Phillip T. Burch $^{2}$. \\ Aaron W. Eckhauser ${ }^{3} \cdot$ Ashwin K. Lal $^{1} \cdot$ Kimberly M. Molina $^{1} \cdot$ Zhining Ou $^{4} \cdot$ Angela P. Presson $^{4} \cdot$ Lindsay J. May $^{1}$
}

Received: 23 February 2020 / Accepted: 16 September 2020 / Published online: 1 October 2020

๑) Springer Science+Business Media, LLC, part of Springer Nature 2020

\begin{abstract}
Infants with aortic coarctation may present with left ventricular (LV) dysfunction which may complicate the postoperative course and lead to increased healthcare costs. We aimed to define the prevalence of moderate to severe left ventricular (LV) systolic dysfunction, evaluate time to recovery, and compare health care costs. Single-center retrospective cohort study at a tertiary care hospital was conducted. Infants $<6$ months old at diagnosis with aortic coarctation were identified using surgical codes for coarctation repair between January 2010 and May 2018. Moderate to severe dysfunction was defined as ejection fraction $(\mathrm{EF})<40 \%$. Of 160 infants studied, 18 (11\%) had moderate to severe LV dysfunction at presentation. Compared to those with better LV function, infants with moderate to severe LV dysfunction were older at presentation (12 vs. 6 days, $p=0.004$ ), had more postoperative cardiac intensive care unit (ICU) days ( 5 vs. $3, p<0.001)$, and more ventilator days ( 3.5 vs. $1, p<0.001)$. The median time to normal LV EF ( $\geq 55 \%)$ was 6 days postoperatively (range 1-230 days). Infants presenting with moderate to severe LV dysfunction had higher index hospitalization costs $(\$ 90,560 \mathrm{vs.} \$ 59,968, p=0.02)$, but no difference in cost of medical follow-up for the first year following discharge $(\$ 3,078 \mathrm{vs}$. $\$ 2,568, p=0.46)$. In the current era, $>10 \%$ of infants with coarctation present with moderate to severe LV dysfunction that typically recovers. Those with moderate to severe dysfunction had longer duration of mechanical ventilation and postoperative cardiac ICU stays, likely driving higher costs of index hospitalization.
\end{abstract}

Keywords Aortic coarctation $\cdot$ Heart failure $\cdot$ Health care cost $\cdot$ Pediatric cardiology

\section{Introduction}

Coarctation of the aorta occurs in about 1 per 2900 live births [1]. In the absence of other complex heart disease coarctation is usually diagnosed postnatally and the infant may present with a wide constellation of findings [2]. As the patent ductus arteriosus (PDA) closes and the narrowing of

Carol A. McFarland

carol.mcfarland@hsc.utah.edu

1 Department of Pediatrics, Division of Pediatric Cardiology, University of Utah, Salt Lake City, UT 84113, USA

2 Department of Surgery, Pediatric Cardiothoracic Surgery, Cook Children's Hospital, Fort Worth, USA

3 Department of Surgery, Division of Pediatric Cardiothoracic Surgery, University of Utah, Salt Lake City, UT, USA

4 Division of Epidemiology, Department of Internal Medicine, School of Medicine, University of Utah, Salt Lake City, UT, USA the aorta progresses, ventricular afterload increases leading to poor left ventricular (LV) systolic function, heart failure, and even cardiogenic shock in a subset of patients [3, 4]. The prevalence of LV dysfunction and clinical outcomes of these infants is not well characterized and data regarding perioperative morbidity and mortality are conflicting [5-7]. In general, children with congenital heart disease who develop heart failure have prolonged inpatient stays, high rates of readmission, and greater inpatient and outpatient costs, but these factors have not been studied in the population of infants with coarctation [8]. To better understand this patient cohort and enable more accurate counseling at the time of diagnosis, we sought to: (1) define the prevalence of moderate to severe LV dysfunction among infants presenting with aortic coarctation in the current era, (2) determine the time to recovery of LV systolic function, and (3) compare the inpatient and outpatient clinical courses and healthcare resource utilization between the group of infants with moderate to severely reduced LV systolic function versus the 
group with normal or only mildly reduced LV function at presentation.

\section{Methods}

This single-center retrospective longitudinal cohort study included infants $<6$ months old diagnosed with coarctation of the aorta in the absence of other complex heart disease who underwent surgical repair between January 2010 and May 2018. The institutional database at Primary Children's Hospital, a tertiary care center, was queried using surgical codes for coarctation repair with or without arch reconstruction. Infants with a PDA, a small atrial or ventricular level shunt, a single anomalous pulmonary vein and those with minor valve abnormalities were included. Infants with large septal defects or other hemodynamically significant associated cardiac lesions were excluded.

Records were reviewed for demographics, pre- and postoperative hospital course, details of surgery, pre- and postoperative echocardiographic data, clinical course from discharge to 1 year postoperatively, and health care costs during the index surgical hospitalization as well as inpatient and outpatient costs from discharge to 1 year after surgery.

Echocardiographic assessment of LV systolic function at the time of diagnosis was used to divide patients into 2 groups using the highest tiered function assessment available within the following hierarchy: ejection fraction (EF) from the 4-chamber view, EF from the 2-chamber view, shortening fraction (SF) using M-mode, or subjective assessment as recorded on the written report. Infants were categorized as having moderate to severely reduced LV systolic function if the $\mathrm{EF}$ was $<40 \%$ or the SF was $<20 \%$ or the reader reported a subjective assessment of moderately or severely reduced LV systolic function [9]. All other infants were categorized as having normal to mildly reduced LV function. For infants in the group with moderate to severely reduced LV function, records were further reviewed for echocardiographic data at hospital discharge and 4 follow-up visits as available (approximately 3 months, 6 months, 9 months and 12 months after hospital discharge). Recovery of LV systolic function was defined as $\mathrm{EF} \geq 55 \%$ or $\mathrm{SF} \geq 28 \%$.

Direct costs were obtained from the hospital. This included inpatient and outpatient costs, and incorporated personnel, facility, and supply costs. Cost of professional fees were not available. All cost values were adjusted for geographic area and for inflation to 2018 dollars using the All Urban Consumers Consumer Price Index-All Items [10].

Primary Children's Hospital and the University of Utah Institutional Review Boards approved this study with a waiver of consent.

\section{Statistical Analysis}

Demographics and clinical outcomes were summarized using median and interquartile range (IQR) for continuous variables due to distribution skew, and counts and percentages for categorical variables. Comparisons between the normal or mildly reduced function group and the moderate to severely reduced function group were performed using nonparametric Wilcoxon rank sum tests, and chi-squared or Fisher's exact tests for categorical variables as appropriate. The $95 \%$ confidence interval for prevalence was calculated using the Clopper-Pearson method for binomial proportion. Univariable logistic regression was performed to determine factors associated with moderate to severely decreased function at presentation. As only 1 factor had a $p$-value $<0.1$, no multivariable regression was performed. A univariable Cox proportional hazards regression model was fit to evaluate the association between age at presentation, and time to normalization of systolic function following surgery within the moderate to severely decreased function group. We were not able to fit a multivariable model due to the small number of patients experiencing moderate to severely decreased function in our cohort. Hazard ratios (HR) and 95\% confidence intervals (CI) were reported. We used a cumulative incidence plot to illustrate the recovery trajectory of systolic function following surgery. Statistical significance was assessed at the 0.05 level and all tests were two-sided. Statistical analyses were performed using R v.3.6.0 [11].

\section{Results}

Of the 163 infants who met study criteria, 3 had no documented evaluation of LV function on the initial echocardiogram and were excluded (Fig. 1). Of the remaining 160 infants who formed the study cohort (Table 1), 18 (11\%, CI: 7, 17\%) had moderate to severely reduced LV systolic function at initial presentation. Gender $(p=0.87)$ and weight at presentation $(p=0.66)$ were similar between groups. No infant in the moderate to severe LV dysfunction cohort was prenatally diagnosed vs. 20 (14\%) in the comparison cohort. Compared to those with better LV function, infants with moderate to severe LV dysfunction were older at presentation ( 12 vs. 6 days old; $p=0.004$ ). There was no difference in cardiopulmonary bypass time $(p=0.45)$ or frequency of median sternotomy for surgical approach $(p=0.31)$ between the groups. In univariable regression analysis, the odds of having moderate to severely reduced function at presentation were lower in the presence of an atrial shunt (Table 2). No other factors were found to have an association with moderate to severely reduced function at presentation. 


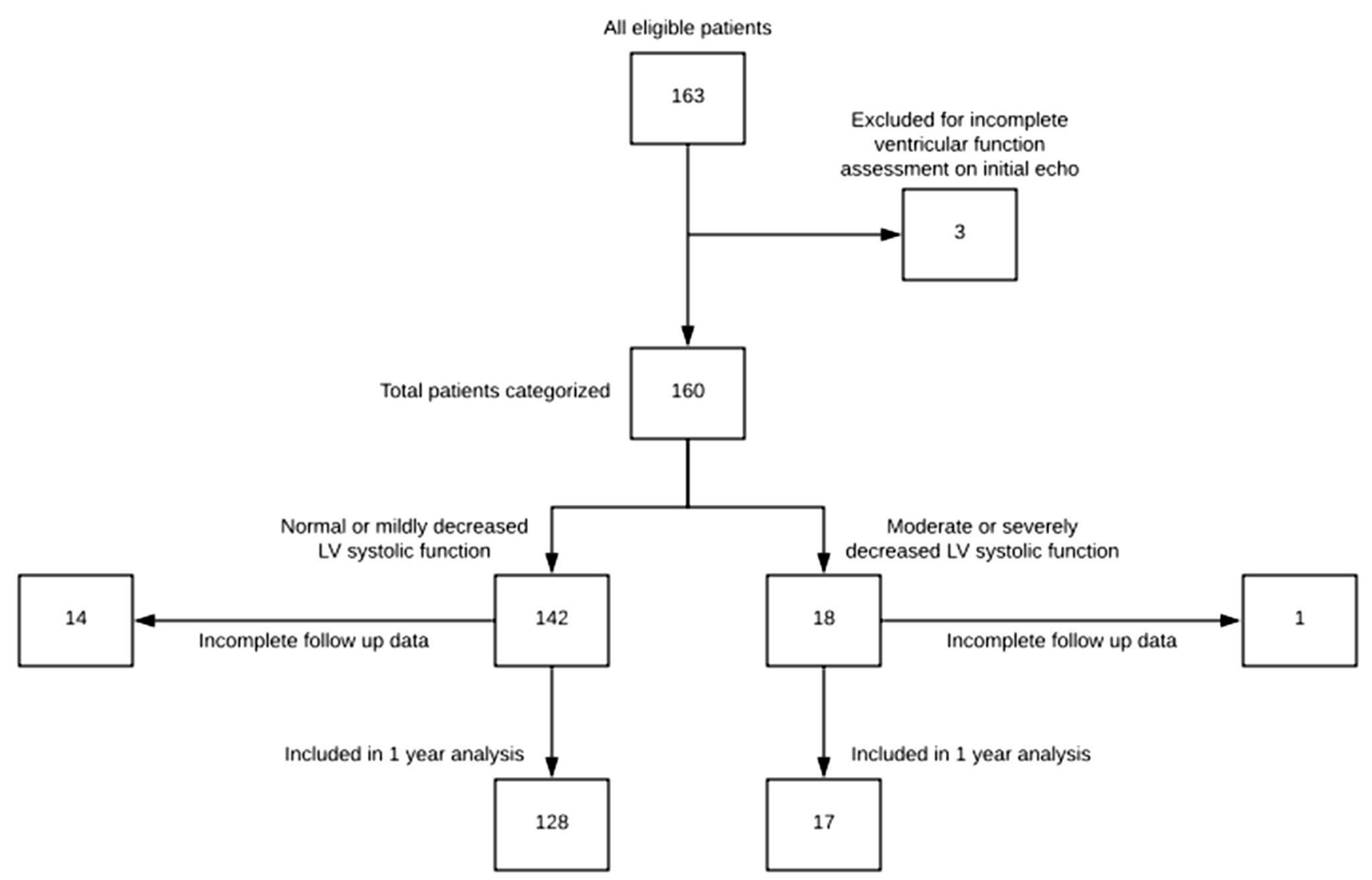

Fig. 1 Patient classification and follow-up diagram

Table 1 Summary of demographics and clinical characteristics at diagnosis

\begin{tabular}{|c|c|c|c|}
\hline & $\begin{array}{l}\text { Moderate or severely decreased function } \\
\text { Median (IQR) or N }(\%) ; N=18\end{array}$ & $\begin{array}{l}\text { Normal or mildly decreased function } \\
\text { Median (IQR) or } \mathrm{N}(\%) ; N=142\end{array}$ & $p$-value \\
\hline Age at diagnosis (days) & $12.2(9.1,27.4)$ & $6.1(0.0,18.3)$ & 0.004 \\
\hline Male sex & $12(67 \%)$ & $92(65 \%)$ & 0.87 \\
\hline Weight at presentation $(\mathrm{kg})$ & $3.5(3.0,3.7)$ & $3.5(3.0,4.1)$ & 0.66 \\
\hline Genetic diagnosis $(\mathrm{Y})$ & $1(6 \%)$ & $16(11 \%)$ & 0.70 \\
\hline Prenatal diagnosis $(\mathrm{Y})$ & $0(0 \%)$ & $20(14 \%)$ & 0.13 \\
\hline $\mathrm{EF}$ at diagnosis & $32.0(25.5,36.0)$ & $65.0(59.0,73.0)$ & $<0.001$ \\
\hline LVIDd z-score at presentation & $0.2(-1.1,3.3)$ & $-1.3(-2.5,-0.4)$ & 0.012 \\
\hline Surgical approach & & & 0.31 \\
\hline Sternotomy & $10(56 \%)$ & $61(43 \%)$ & \\
\hline Thoracotomy & $8(44 \%)$ & $81(57 \%)$ & \\
\hline \multicolumn{4}{|l|}{ Concomitant operations with initial surgery } \\
\hline ASD closure* & $5(28 \%)$ & $17(12 \%)$ & 0.07 \\
\hline Cardiopulmonary bypass time (minutes) & $78.0(0.0,94.5)$ & $0.0(0.0,94.0)$ & 0.45 \\
\hline
\end{tabular}

$E F$ ejection fraction, $L V I D d$ left ventricular internal dimension in diastole, $A S D$ atrial septal defect

${ }^{*}$ Small ASDs were closed at the discretion of the surgeon

Patient outcomes and healthcare resource utilization patterns are summarized in Table 3. Those with moderate to severe LV dysfunction had more postoperative cardiac intensive care unit (ICU) days ( 5 vs. 3 days, $p<0.001$ ), and more ventilator days $(3.5$ vs. 1 day, $p<0.001)$. They were also more likely to have $\geq 3$ postoperative echocardiograms 
Table 2 Univariable regression of risk factors for moderate to severely decreased function

\begin{tabular}{llll}
\hline & Odds ratio & $95 \%$ CI & $p$-value \\
\hline Age at presentation & 1.16 & $0.72,1.71$ & 0.49 \\
Weight at presentation & 0.94 & $0.56,1.55$ & 0.80 \\
Male gender & 1.09 & $0.4,3.28$ & 0.87 \\
Genetic diagnosis & 0.46 & $0.02,2.49$ & 0.46 \\
Presence of ASD/PFO & 0.25 & $0.09,0.81$ & 0.02 \\
\hline
\end{tabular}

$C I$ confidence interval, $A S D$ atrial septal defect, $P F O$ patent foramen ovale

while in the hospital than those with normal or mildly reduced function $(17 \%$ vs. $3 \%, p=0.03)$. The total hospital length of stay was similar between the moderate to severe LV dysfunction group and the comparison group $(p=0.34)$. Prior to discharge, 1 infant from the moderate to severe LV dysfunction group had a bradycardic arrest on postoperative day 1 and was placed on extracorporeal membrane oxygenation (ECMO), but was successfully decannulated after 5 days. No infants from the normal to mildly reduced LV function group required mechanical circulatory support. Postoperative complications were rare in this cohort of patients and there was no difference between the normal to mildly reduced function group and the moderate to severely reduced function group.

The median time to a documented normal LV EF ( $\geq 55 \%)$ was 6 days postoperatively (Fig. 2; range 1-230 days) for those that presented with moderate to severely reduced LV function. Data were available at 1-year follow-up for 145 infants: 1 from the moderate to severely reduced function group and 14 from the group with normal to mildly reduced function had incomplete data. The LV EF was normal by 1-year follow-up for the entire cohort with 1-year data. Age at presentation was not associated with time to normalization of $\mathrm{EF}$ (hazard ratio 0.6, 95\% CI: 0.3, 1.2). Of those with 1-year follow-up, $24 \%$ with moderate to severe LV dysfunction had a catheter or surgical based reintervention vs. $12 \%$ $(p=0.43)$ in the comparison cohort. The majority of patients

Table 3 Outcomes and healthcare utilization at 1-year follow-up

\begin{tabular}{|c|c|c|c|}
\hline & $\begin{array}{l}\text { Moderate or severely decreased } \\
\text { function Median (IQR) or } \mathrm{N}(\%) \\
N=18\end{array}$ & $\begin{array}{l}\text { Normal or mildly decreased } \\
\text { function Median (IQR) or N (\%) } \\
N=142\end{array}$ & $p$-value \\
\hline Mortality & $0(0 \%)$ & $2(1 \%)$ & 0.76 \\
\hline Transplant & $0(0 \%)$ & $0(0 \%)$ & $>0.99$ \\
\hline Mechanical ventilation (days) & $3.5(2.2,6.0)$ & $1.0(1.0,3.0)$ & $<0.01$ \\
\hline Preoperative cardiac ICU length of stay (days) & $2.0(1.2,3.8)$ & $3.0(2.0,6.0)$ & 0.12 \\
\hline Postoperative cardiac ICU length of stay (days) & $5.0(3.2,7.0)$ & $3.0(2.0,4.0)$ & $<0.01$ \\
\hline Hospital length of stay (days) & $12.5(10.0,17.8)$ & $10.0(7.0,16.0)$ & 0.34 \\
\hline \multicolumn{4}{|l|}{ Postoperative complications } \\
\hline Dialysis & $0(0 \%)$ & $0(0 \%)$ & $>0.99$ \\
\hline Stroke & $0(0 \%)$ & $0(0 \%)$ & $>0.99$ \\
\hline Chylothorax & $0(0 \%)$ & $4(3 \%)$ & $>0.99$ \\
\hline Infection & $0(0 \%)$ & $3(2 \%)$ & $>0.99$ \\
\hline $\begin{array}{l}\text { \# of infants with } \geq 3 \text { echoes obtained during postsurgery } \\
\text { hospital course }\end{array}$ & $3(17 \%)$ & $4(3 \%)$ & 0.03 \\
\hline $\begin{array}{l}\text { \# of infants with } \geq 3 \text { echoes obtained during first } 12 \text { months } \\
\text { following hospital discharge }\end{array}$ & $3(19 \%)$ & $11(9 \%)$ & 0.20 \\
\hline $\begin{array}{l}\text { \# of Pediatric Cardiology Clinic visits in first } 12 \text { months } \\
\text { following hospital discharge }\end{array}$ & $3.0(3.0,4.0)$ & $3.0(3.0,4.0)$ & 0.14 \\
\hline $\begin{array}{l}\text { Hospital readmissions } \geq 1 \text { during first year following dis- } \\
\text { charge }\end{array}$ & $4(22 \%)$ & $22(15 \%)$ & 0.06 \\
\hline Cardiac* & $1(25 \%)$ & $7(32 \%)$ & \\
\hline Noncardiac + & $3(75 \%)$ & $15(68 \%)$ & \\
\hline Hospitalization cost (\$) & $90560(63485,121532)$ & 59968 (34 320, 92 813) & 0.02 \\
\hline Costs between discharge and 1-year follow-up (\$) & $3078(1329,15276)$ & $2568(796,8365)$ & 0.46 \\
\hline
\end{tabular}

ICU intensive care unit

* Cardiac reasons for readmission included reintervention, and need for IV antibiotics in the setting of a sternal wound infection

+ Noncardiac reasons for readmission included ENT procedures, increased work of breathing/bronchiolitis, GI/GU procedures, dehydration/ emesis, and new onset diabetes mellitus 


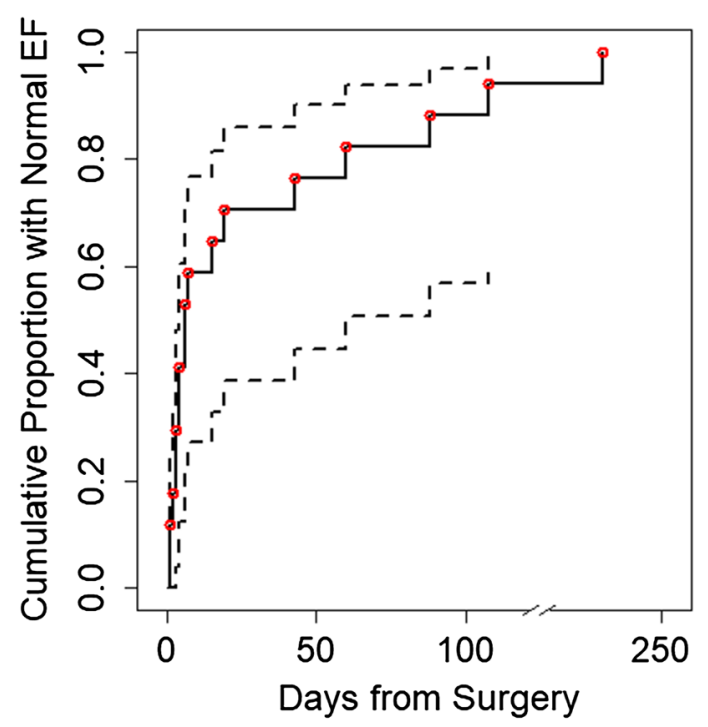

Fig. 2 Kaplan-Meier curve for time to normal left ventricular ejection fraction (EF). The solid line represents the cumulative proportion of patients with normal $\mathrm{EF}$ who presented with moderate to severely decreased LV function. The dashed lines represent $95 \%$ confidence intervals. Circles represent time of first documented normal EF

who required reintervention underwent balloon angioplasty of the aorta although 1 infant (25\%) from the moderate to severely reduced function group and $2(14 \%)$ from the group with normal to mildly reduced function had a surgical reintervention. There was no difference in frequency of being readmitted 1 or more times within 1 year between the groups $(p=0.06)$. Within the first year, no patient in either group was listed for heart transplant. No deaths occurred in the moderate to severe LV dysfunction group, but 2 infants (both with genetic abnormalities) died in the comparison group.

Infants presenting with moderate to severe LV dysfunction had higher index hospitalization costs $(\$ 90,560$ vs. $\$ 59,968, p=0.02$ ), but there was no difference in cost of medical follow-up for the first year following discharge $(p=0.46)$. The likelihood of having $\geq 3$ echocardiograms in the year after hospital discharge was not statistically different between groups $(p=0.2)$.

\section{Discussion}

Our study is the first to define the prevalence of moderate to severe LV dysfunction among infants presenting with coarctation, and to systematically characterize their clinical course and healthcare utilization in the current era. Moderate to severely reduced LV systolic function was not infrequent, affecting $11 \%$ of infants with coarctation. Those presenting with poor LV systolic function were older, required more support following surgery, and had higher index hospitalization costs. These findings are not surprising and are likely explained by longer exposure of the LV to the high afterload that results from aortic arch obstruction. However, older age at presentation may also be related to delayed closure of the PDA. Nevertheless, all patients in this cohort had normal LV systolic function within 1 year from surgery and many recovered LV function within 1 week of surgery, providing encouraging information when counseling parents about short- and long-term expectations.

Patients with LV systolic dysfunction in other populations are at higher risk for hospital readmission, and undergo more frequent clinic visits and imaging, increasing the economic and social burden on both the families and the medical system [12]. In our population, moderate to severely decreased LV function was associated with prolonged support (ventilator and postoperative cardiac ICU days) while in the hospital. This is an important finding as increased ICU length of stay has been associated with worse neurodevelopmental outcomes including IQ in other studies [13]. Infants in the moderate to severe LV dysfunction group also had more echocardiograms during their surgical hospitalization that contributed to higher costs. However, in contrast to other studies, readmissions and cost of medical care between discharge after surgery and 1-year follow-up was similar between the groups, as was the number of echocardiograms, suggesting that serial echocardiograms were performed less frequently after recovery of LV EF. Our data support prior studies suggesting that most infants recover LV function, and that advanced heart failure therapies, such as mechanical circulatory support, are rarely used [14-16].

Early recognition of infants with coarctation, prior to the development of ventricular dysfunction, resulted in lower costs for the surgical hospitalization. Unfortunately, coarctation is a lesion that may still be missed despite mandatory pulse oximetry screening, since oxygen saturations may be normal with little difference between upper and lower extremities. Given this finding, consideration may be given to careful physical examination earlier than the 2-week well baby check that is currently standard practice in the USA.

In addition to the single center retrospective design of this study, there are several other limitations. Due to the relatively small cohort of patients, this study was only powered to detect large differences between the two subgroups. In addition, as the central surgical center for a wide geographic area, our database did not have follow-up data for infants cared for by providers outside of our network, though this affected only a minority of patients. Finally, cost data did not include professional fees and may not represent the entire cost of care. However, given the aim of comparing costs between the two subgroups, this does not detract from the study findings given that professional fees were not included for either subgroup. 
In conclusion, $11 \%$ of infants with coarctation have moderate to severe left ventricular dysfunction at presentation. This was associated with longer postoperative cardiac ICU length of stay and higher costs for the index hospitalization, but the discrepancy in costs did not persist after discharge from the index surgery. Parents of these infants should be counseled that LV function will often recover early after surgery, and generally within the first postoperative year.

Author's Contributions All authors contributed to the study conception and design. Data collection and analysis were performed by Carol McFarland, Zhining Ou and Angela Presson. The first draft of the manuscript was written by Carol McFarland and all authors commented on previous versions of the manuscript. All authors read and approved the final manuscript.

Funding This investigation was supported by the University of Utah Study Design and Biostatistics Center, with funding in part from the National Center for Research Resources and the National Center for Advancing Translational Sciences, National Institutes of Health, through Grant UL1TR002538 (formerly 5UL1TR001067-05, 8UL1TR000105 and UL1RR025764). This research was supported by the National Institutes of Health under Ruth L. Kirschtein National Research Service Award T32HL007576 from the National Heart, Lung and Blood Institute.

Data Availability The datasets generated during the current study are available from the corresponding author on reasonable request.

\section{Compliance with ethical standards}

Conflicts of interest The authors declare that they have no conflict of interest.

Ethics approval Primary Children's Hospital and the University of Utah Institutional Review Boards approved this study with a waiver of consent.

\section{References}

1. Nguyen L, Cook SC. Coarctation of the Aorta: Strategies for Improving Outcomes. Cardiol Clin. 2015;33(4):521-530, vii.

2. Liberman RF, Getz KD, Lin AE et al (2014) Delayed diagnosis of critical congenital heart defects: trends and associated factors. Pediatrics 134(2):e373-381

3. Jashari H, Lannering K, Mellander M, Ibrahimi P, Rydberg A, Henein MY (2017) Coarctation repair normalizes left ventricular function and aorto-septal angle in neonates. Congenit Heart Dis 12(2):218-225

4. Klitsie LM, Roest AA, Kuipers IM et al (2013) Enhanced characterization of ventricular performance after coarctation repair in neonates and young children. Ann Thorac Surg 96(2):629-636

5. McGuinness JG, Elhassan Y, Lee SY et al (2010) Do high-risk infants have a poorer outcome from primary repair of coarctation? Analysis of 192 infants over 20 years. Ann Thorac Surg 90(6):2023-2027

6. Fesseha AK, Eidem BW, Dibardino DJ et al (2005) Neonates with aortic coarctation and cardiogenic shock: presentation and outcomes. Ann Thorac Surg 79(5):1650-1655

7. Kim TH, Shin YR, Kim YS, et al. Central-Approach Surgical Repair of Coarctation of the Aorta with a Back-up Left Ventricular Assist Device for an Infant Presenting with Severe Left Ventricular Dysfunction. In: Korean J Thorac Cardiovasc Surg. Vol 48. Korea (South)2015:407-410.

8. Nandi D, Rossano JW, Wang Y, Jerrell JM (2017) Risk Factors for Heart Failure and Its Costs Among Children with Complex Congenital Heart Disease in a Medicaid Cohort. Pediatr Cardiol 38(8):1672-1679

9. Lang RM, Bierig M, Devereux RB et al (2006) Recommendations for chamber quantification. Eur J Echocardiogr 7(2):79-108

10. All urban consumers consumer price index. Published 2018. Accessed February 2019.

11. R: A language and environment for statistical computing. [computer program]. Vienna, Austria: R Foundation for Statistical Computing; 2019.

12. Almond CS, Rosenthal DN, Wood M, et al. 30-day readmission rate and center variability for US children hospitalized with heart failure. Circulation. 2015;132.

13. Newburger JW, Wypij D, Bellinger DC et al (2003) Length of stay after infant heart surgery is related to cognitive outcome at age 8 years. J Pediatr 143(1):67-73

14. Bello Valls ML, Salih HG, El Dadah OM et al (2018) Cardiac recovery and outcome of neonates and infants presenting with severe aortic coarctation and depressed cardiac function. Egypt Heart J 70(4):255-260

15. Pacileo G, Pisacane C, Russo MG et al (2001) Left ventricular remodeling and mechanics after successful repair of aortic coarctation. Am J Cardiol 87(6):748-752

16. Balderrabano-Saucedo NA, Vizcaino-Alarcon A, Reyes-de la Cruz L, Espinosa-Islas G, Arevalo-Salas A, Segura-Stanford B. [Left ventricular function in children after successful repair of aortic coarctation]. Rev Esp Cardiol. 2008;61(11):1126-1133.

Publisher's Note Springer Nature remains neutral with regard to jurisdictional claims in published maps and institutional affiliations. 